\title{
The changing epidemiology of hepatitis $B$ and $C$ infections in Nanoro, rural Burkina Faso: a random sampling survey
}

\author{
Moussa Lingani ${ }^{1,2,3}$, Tomoyuki Akita', Serge Ouoba ${ }^{3}$, Shintaro Nagashima', Palwende Romuald Boua ${ }^{3}$, \\ Kazuaki Takahashi', Basile Kam³ ${ }^{3}$ Aya Sugiyama' ${ }^{1}$,Théodore Nikiema ${ }^{3}$, Chikako Yamamoto $^{1}$, Athanase Somé3 \\ Karim Derra ${ }^{2,3}, \mathrm{Ko} \mathrm{Ko}^{1}$, Hermann Sorgho ${ }^{2,3}$, Zekiba Tarnagda ${ }^{4}$, Halidou Tinto ${ }^{2,3}$ and Junko Tanaka ${ }^{1 *}$ (D)
}

\begin{abstract}
Background: This study sought to provide up-to-date hepatitis B (HBV) and C (HCV) seroprevalence in rural Burkina Faso decade after hepatitis B vaccine was introduced in the national immunization scheduled for children.

Methods: In 2018, a community-based, random sampling strategy with probability proportional to population size was conducted in Nanoro to investigate the prevalence of viral hepatitis in children and their mothers. Sociodemographic, vaccination history and risk factors were assessed by interview and health books. HBsAg rapid tests were done by finger prick and Dried Blood Spots (DBS) were collected for hepatitis seromarkers by chemiluminescence enzyme immunoassay. Positive samples underwent confirmatory PCR and phylogenetic analysis.

Results: Data were presented on 240 mother-child pairs. HBsAg Prevalence was $0.8 \%$ in children and $6.3 \%$ in mothers. Hepatitis B core antibody positivity was $89.2 \%$ in mothers, $59.2 \%$ in children and was associated with age, sex and scarification. Hepatitis B surface antibodies prevalence was $37.5 \%$ in children and $5.8 \%$ in mothers. Good vaccination coverage was limited by home delivery. Phylogenetic analysis of HBV strains based on full genome sequences $(n=7)$ and s-fragment sequences $(n=6)$ revealed genotype A, E, and recombinant A3/E. Viral genome homology was reported in one mother-child pair. Anti-HCV prevalence was $5.4 \%$ in mothers, $2.1 \%$ in children and strains belonged to genotype 2 .
\end{abstract}

Conclusions: In Nanoro, HBsAg prevalence was low in children, intermediate in mothers and mother-to-child transmission persists. Home delivery was a limiting factor of Hepatitis B vaccination coverage. HBV genotype E was predominant and genotype A3/E is reported for the first time in Burkina Faso.

Keywords: Burkina Faso, Hepatitis B virus, Hepatitis C virus, Genotype, Vaccination

\section{Background}

Although the global burden of viral hepatitis is gradually decreasing worldwide, it still represents an international health challenge particularly in low-and-middle income countries [1]. It is estimated that globally 1.34 million people died from viral hepatitis complications in 2015 [2]. Sub-Saharan African countries carry a heavy burden

\footnotetext{
* Correspondence: jun-tanaka@hirosima-u.ac.jp

${ }^{1}$ Department of Epidemiology, Infectious Disease Control and Prevention, Hiroshima University Graduate School of Biomedical and Health Sciences, 1-2-3 Kasumi, Minami-ku, Hiroshima 734-8551, Japan

Full list of author information is available at the end of the article
}

with over 60 million people living with hepatitis B (HBV) among whom, 2 million live in Burkina Faso [3, 4]. According to recent estimates, hepatitis B causes $54 \%$ of all liver cancer cases worldwide [5-7]. Meanwhile, hepatitis $\mathrm{C}$ virus (HCV) causes 10 to $25 \%$ of all liver cancers worldwide and affects 10-30 million people in West Africa [7-9]. Despite the introduction of hepatitis $B$ vaccine into Burkina Faso's national expanded program on immunization (EPI) for children in 2006, related death has increased and cost 2600 lives in 2015 [10]. The picture could even be worse given that accurate identification of death causes in sub-Saharan Africa

(c) The Author(s). 2020 Open Access This article is distributed under the terms of the Creative Commons Attribution 4.0 International License (http://creativecommons.org/licenses/by/4.0/), which permits unrestricted use, distribution, and 
is still a challenge [11]. In African regions, transmission is reported to be mainly perinatal through mother to child transmission or horizontal transmission through exposure to infected body fluids $[12,13]$.

Elimination of hepatitis B infection can achieve in a particular country when national government started to develop and implement efficacious strategies including free children vaccination against HBV, case detection and treatment with effective drugs [12,13]. Elimination of $\mathrm{HCV}$ is also envisioned since highly effective direct antiviral agents (DAA) are now available in low-income countries and are reported to cure infection within 10 to 12 weeks of treatment $[14,15]$.

Since 2006, universal vaccination against HBV was started as part of Expanded Programme of Immunization (EPI) but Hepatitis B birth dose is not recommended to all new born till now. Specific to HBsAg positive mother, new guideline was recently released in September, 2019 in which all HBsAg positive mothers except those with HBV DNA < $2000 \mathrm{IU} /$ $\mathrm{ml}$ should be treated with Tenofovir (TDF) and the birth dose should be given to the baby. Burkina Faso developed a 5-years strategic plan in 2017 to guide efforts based on improving vaccine coverage in infants, supply of effective antiviral drugs, and emphasizing on raising awareness. However, detail epidemiological data to support these efforts is still limited as well as evidence of the impact of current interventions $[10,16]$. A severe limitation in this respect is the scarcity of reliable prevalence data from population-based studies. A recent meta-analysis reported an overall HBV prevalence of $9.4 \%$ in the country with however important regional variations and large estimation intervals [3]. These variations could be related to the characteristics of the diagnostic tests used and the specific populations screened like blood donors, pregnant women or HIV infected people. A recently published study reported a nationwide prevalence of 7.8 and $3.2 \%$ respectively for $\mathrm{HBV}$ and HCV among women with data collected since 2010 in HIV negative adult population [16]. To this regard, more recent community-level data is still needed to evaluate the impact of interventions in the rural area and subsequently develop strategies for improvement. Using HemaSpot ${ }^{\mathrm{m}}$ (Spot on Sciences, USA) blood collection device, we investigate HBV and HCV infections seroprevalence and risk factors. Genotypic characterization has been performed, and key risk factors among children and their mothers living in Nanoro, at top north of centre west region of Burkina Faso were investigated. Our population-based study is expected to provide updated and additional information for adequate planning of preventive measures against viral hepatitis infections.

\section{Methods}

\section{Study design, site and participants}

This was a cross-sectional survey conducted among children born after 2006 and their respective mothers in Nanoro health and demographic surveillance system area (HDSS), Centre-west region of Burkina Faso. The study site covers an updated population size of 63,000 inhabitants. The study area was described elsewhere [17]. Nanoro is a rural area located in the Centre-West of the country. In this area, health care is provided by seven peripheral health posts and one referral hospital. Based on an anticipated hepatitis B surface antigen (HBsAg) prevalence in the rural area of $17.3 \%$ in Burkina Faso [3], confidence level of 95\%, precision of 5, and $10 \%$ maximum missing data, the minimum sample size for mothers is 240 participants. As for children required sample size is less than 240, because the prevalence among children is thought as lower than that among adults. Therefore, the required sample size was totally 240 pairs of children and their mothers. The study on HBsAg prevalence in rural area of Burkina Faso is limited. The study area was randomly selected among all rural area of Burkina Faso. This study population covers $0.46 \%$ of all rural population in Burkina Faso. All 24 villages of the Nanoro HDSS were selected. We conducted sampling procedure as follows: First mothers were selected by random sampling with probability proportional to village size. Second, children were selected using simple random sampling from children of selected mothers when mother have more than one eligible child. Then, a list of 240 pairs of mothers above 15 years old and their child was generated from the HDSS database. Selected potential participants were visited at home by study investigators. Informed consent was obtained before study related activities were performed. If the woman has more than an eligible child aged one to 11 years old, we selected the participant (child) among eligible children using an automatic random number generator (RNG).

\section{Questionnaire survey}

Trained study investigators administered a standardized questionnaire to all study participants (Additional file 1: Appendix 1a and 1b). Socio-demographic data such as age, sex, ethnic group, educational level, occupation, marital status, vaccination history; and potential risk factors including surgery, blood transfusion, injections, tattoos, skin-piercings, scarification, birthplace, were collected. The vaccination history was taken mainly from vaccination card. If the child has no vaccination card, the information was obtained by the recall-memory of their mothers. For the mother, the information could only be obtained by their recall memory. 


\section{Serological and molecular assays}

A rapid point of care Alere Determine ${ }^{\mathrm{Tm}} \mathrm{HBsAg}$ test strips, sensitivity $95-100 \%$ and specificity 96-100\% (Abbott Japan Co., Ltd.) using 1 drop $(20 \mu \mathrm{l})$ was performed using blood obtained from participants finger pricks. Completely vaccinated referred to children who received 3 doses of pentavalent vaccine. Three drops of blood $(60 \mu \mathrm{l})$ were collected on HemaSpot ${ }^{\mathrm{Tm}}$ sampling devices, dried and stored at minus $80^{\circ} \mathrm{C}$ in Nanoro and shipped to Hiroshima university for laboratory analysis. The

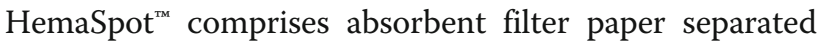
into eight fragments in a fan-shape with a central hole. Three fragments were detached and eluted at room temperature in $600 \mu \mathrm{L}$ of elution buffer [Tris-buffered saline (TBS): $50 \mathrm{mM}$ Tris, $150 \mathrm{mM} \mathrm{NaCl}, 0.1 \%$ Proclin 300 and $0.05 \%$ Tween 20 at pH 7.2]. The mixtures were stirred in shaking plates for 1 hour and subsequently centrifuged at $12000 \mathrm{rpm}$ for $5 \mathrm{~min}$ at $4{ }^{\circ} \mathrm{C}$ and the supernatants were separated. Using Lumipulse G1200 (Fujirebio Inc. Inc., Japan), a chemiluminescent enzyme immunoassay (CLEIA) was used to detect HBsAg (Lumipulse $^{\oplus}$-II HBsAg, Fujirebio Inc., Japan with reported sensitivity of $100 \%$ and specificity of $99.7 \%$ [18]) with a cut-off index (COI) of 1.0, HBcAb (Lumipulse ${ }^{\oplus} \mathrm{HBcAb}-$ $\mathrm{N}$, Fujirebio Inc., Japan, with reported sensitivity of $88.4 \%$ and specificity of $95.2 \%$ [18]) COI of 0.6, HBsAb (Lumipulse ${ }^{\oplus}$ HBsAb-N, Fujirebio Inc., Japan, with reported sensitivity of $95 \%$ and specificity of $100 \%$ [18]) COI of 3.4, HBeAg (Lumipulse ${ }^{\oplus}$ I HBeAg, Fujirebio Inc., Japan, with reported sensitivity of $97.6 \%$ and specificity of $98.2 \%$ [18]) COI of 1.0, HBeAb (Lumipulse ${ }^{\circ}$ HBeAb$\mathrm{N}$ Inc., Fujirebio, Japan, with reported sensitivity of $100 \%$ and specificity of $95.9 \%$ [18]) COI of 50.0. We considered the positivity of $\mathrm{HBsAg}$ or $\mathrm{HBcAb}$ sero-positivity as indicative of "HBV exposure". Samples positive to $\mathrm{HBsAb}$ and negative for both HBsAg and $\mathrm{HBcAb}$ were classified as "serologically vaccinated". Those with negative test results to $\mathrm{HBsAg}, \mathrm{HBsAb}$ and $\mathrm{HBcAb}$ were designated as "susceptible" and at risk of HBV infection. To investigate HCV infection, HCV antibody (HCVAb) positivity (LumipulseII ${ }^{\circ}$ Ortho- $\mathrm{HCV}$, Ortho Clinical Diagnostics, Japan) with a cut-off value of 0.6 was measured using CLEIA.

\section{Molecular assay and genome sequencing}

In HBsAg positive samples, confirmatory tests were performed to detected and quantify HBV DNA by nucleic acid amplification test (NAT). At first, nucleic acid was extracted from HemaSpot ${ }^{\text {TM }}$ using SMITEST EXR\&D (Medical and Biological Laboratories co., LTD, MA, USA). To detect HBV DNA, nested PCR assays with Applied Biosystems MiniAmp Plus Thermal Cycler (Thermo Fisher Scientific, Tokyo, Japan) were performed using primers obtained from Minegishi $\mathrm{K}$ et al work
[19]. For viral load assessment, nucleic acid extracts were amplified and measured using a real-time PCR TaqMan Fast Universal PCR Master Mix (2X); Applied Biosystems, StepOne $^{\mathrm{Tm}}$ (Thermo Fisher Scientific, Tokyo, Japan) with primers and probe from HBV s-region gene $[20,21]$. Hepatis $C$ virus RNA was also detected using nested PCR amplification with in-house designed primer set from the conserved core region gene.

Genome sequencing for genotype analysis was conducted. To this regard, PCR products were generated using PrimeStar GXL (Takara Bio Inc., Shiga, Japan). Subsequently, a direct BigDye Terminator v3.1 Cycle Sequencing Kit; (Applied Biosystems, Foster city, CA, USA) was used.

\section{Data management and statistical analysis}

Study data were collected and managed using REDCap electronic data capture tools hosted at the Clinical Research Unit of Nanoro (CRUN). Data were analysed using Stata, Version 15 (StataCorp. 2017, TX). Proportions were estimated with $95 \%$ exact confidence intervals $(95 \% \mathrm{CI})$. The $\mathrm{X}^{2}$ or Fisher's exact test were used as appropriate to compare proportions between groups. For risk factors of HBsAg positivity, $\mathrm{HBV}$ exposure and $\mathrm{HBcAb}$ positivity in children and mothers, crude oddratios (OR), adjusted OR and their 95\% CIs were calculated by a univariate logistic regression and multivariate logistic-regression using a stepwise selection procedure (method of increasing and decreasing based on $p$-value threshold: both of probability to enter and probability to leave are 0.3) Covariate for analysis in children are age group, one pentavalent at least, completely vaccine, gender, ethnic, blood transfusion, birth assistance and traditional scarification. Covariate for analysis in mothers are age group, occupation, partner occupation, schooling status, birth place, pregnancy, genital mutilation, marital status, birth assistance, know about MTCT and Heard about hepatitis. $P$-value $<5 \%$ was considered statistically significant.

\section{Ethical considerations}

The study protocol was approved by the ethics committee for epidemiological research of Hiroshima University (Certificate E-1257) and the ethics committee of the ministry of health in Burkina Faso (Deliberation CERS2018-02-023). All participants signed an informed consent before they can enter the study. Participants tested positive to hepatitis infection were referred to a specialized centre.

\section{Results}

\section{Demographic characteristics of study participants}

A total of 480 respondents including 240 children (born between 2007 and 2016) and 240 mothers were included 
in the study. Mothers average age was 33.2 \pm 7.8 years, with majority of them were farmers (93.3\%) and $9.2 \%$ of them were pregnant. Children median age was 4.0 years (53.3\% males) and $89.2 \%$ of them were born in healthcare facilities. Table 1 summarize the seromarkers positivity in relation to study population background's characteristics.

\section{Prevalence of hepatitis B infection based on the questionnaire survey}

By rapid diagnosis test, HBsAg prevalence among children was $0.8 \%(2 / 240)$ and $5.8 \%(14 / 240)$ among mothers. One sample indeterminate by rapid diagnosis test, showed a positive result in the laboratory testing. The Cohen's Kappa coefficient ( $\mathrm{k}$ ) showed a very good degree of agreement between the rapid testing and the CLEIA method (0.97; 95\%CI, 0.91-1.00).

From the questionnaire, $11.7 \%$ (28/240, 95\% CI, 7.916.4) of mothers were aware of the existence of the hepatitis B vaccine. Sixty-three percent 63.0\% (151/240, 95\% CI, 56.4-69.0) of children received 3 doses of pentavalent vaccine. The proportion of completely vaccinated children was significantly higher in the under 5-year-old children than the older children $(75.4 \%$ vs $47.2 \%, p<$ 0.001 ) while none of their mothers was completely vaccinated in this survey.

\section{Serological survey \\ Prevalence of HBV infection based on DBS}

In Total, 6.3\% (95\% CI, 3.5-10.1) of mothers, 0.8\% (95\% CI, $0.1-3.0$ ) of children, $8.7 \%$ (95\% CI, 1.1-28.0) of pregnant women and $6.7 \%$ (95\% CI, 0.2-31.2) of children born to a positive HBsAg mother were tested positive for HBsAg (Fig. 1a). Approximately 89.2\% (95\% CI, 84.5-92.8) of mothers and 59.2\% (95\% CI, 52.6-65.4) of children were classified as exposed (HBsAg / HBcAb positive) (Additional file 2: Appendix 2). Risk analysis showed significant higher risk of infection in over 5-year old children $(\mathrm{aOR}=2.1,95 \% \mathrm{CI}, 1.2-3.8, p=0.008)$, in female $(\mathrm{aOR}=1.7,95 \% \mathrm{CI}, 1.1-2.9, p=0.047)$ and in children with traditional scarification $(\mathrm{aOR}=2.7,95 \% \mathrm{CI}$, 1.1-6.7, $p=0.038$ ) (Tables 2 and 3). High viral replication marker (HBeAg positive) was observed in $0.8 \%$ (95\%CI, $0.1-3.0)$ of children and $1.3 \%$ (95\%CI, 0.3-3.6) mothers (Fig.1b). In HBsAg carrier mothers, HBeAb, a surrogate for reduced viral replication, was positive in $60.0 \%$ (95\% CI, 32.3-83.6\%) of infected mothers while none of the children was positive (Fig. 1b).

\section{Survey on vaccine effects}

Among 240 mother-child pairs, only one mother had received hepatitis B vaccine for two times while $63.0 \%$ of children (151/240) had received complete 3 doses hepatitis B vaccine and $66.3 \%$ of children (159/240) had received at least 1 dose of hepatitis B vaccine. Approximately $5.8 \%$ of mothers and $37.5 \%$ of children had protective level of HBsAb (Additional file 2: Appendix 2). Among mothers, all were due to past infections while $60 \%$ of protected children were also secondary to past infections. Only $15.0 \%$ (95\% CI, 10.7-20.1) of all children were immune by vaccination. This proportion of vaccine protected children was significantly higher in children born within health centres $(p=0.042)$. Even though this was not statistically significant in multivariate analysis, a higher trend of protected children was observed in children born in health facilities than those born at home (aOR $=0.4,95 \%$ CI $0.1-1.1, p=0.073$ ).

\section{Hepatitis B surface antigen positive samples}

Additional file 3: Appendix 3 summarises the characteristics of samples with positive $\mathrm{HBsAg}$ test results. HBV DNA was detected in $76.5 \%$ (13/17) including all positive children. The titter of HBV DNA was measured in 11 samples and viral load ranged from 5 copies/mL to $6.1 \mathrm{E}+$ 07 copies/mL. Genotyping was successful in 13 samples, and HBV genotype E was predominant with $61.5 \%$ (8/13), followed by genotype A with $23.1 \%(3 / 13)$ and a recombinant genotype A/E 15.4\% (2/13). The full length genome sequencing was completed in 7 samples. Case B18-025 (child) and case B18-026 (her mother) had 100\% identical genetic sequences. After, phylogenetic analysis and comparison of the strain sequences with sequence in GenBank it was found that the reported genotype A/E was close to A3/E (GQ161753) Ghana and the genotype E strain 18-025-BUR, 18-200-BUR, and 18-456-BUR close to sequence identify in the western part of the country in Bobo-Dioulasso (Fig. 2) [22].

\section{Prevalence of HCV infection}

$\mathrm{HCV}$ antibodies were positive among 5.4\% (95\% CI, 2.9-9.1) of mothers and $2.1 \%$ (95\% CI, 0.7-4.8) of children. Virus RNA was tested and detected in one positive sample. Phylogenetic analysis reported hepatitis $\mathrm{C}$ genotype 2 (Fig. 3). Difference in anti-HCV rate was significant between the participant ethnic group in mothers $(p=0.045)$. By multivariate analysis in mother, history of blood transfusion yield OR of 6.2 (95\%CI, 0.6-64.3) but it has no significant association to $\mathrm{HCV}$ infection $(p=0.125)$.

\section{Discussion}

In this study, we investigated the prevalence and risks factors of hepatitis $B$ and hepatitis $C$ virus infections among children born after hepatitis B vaccine was introduced in the national EPI schedule and in their respective mothers. The study was conducted in Nanoro, a rural area of the centre-west region of Burkina Faso and a random sampling method was used to select a 


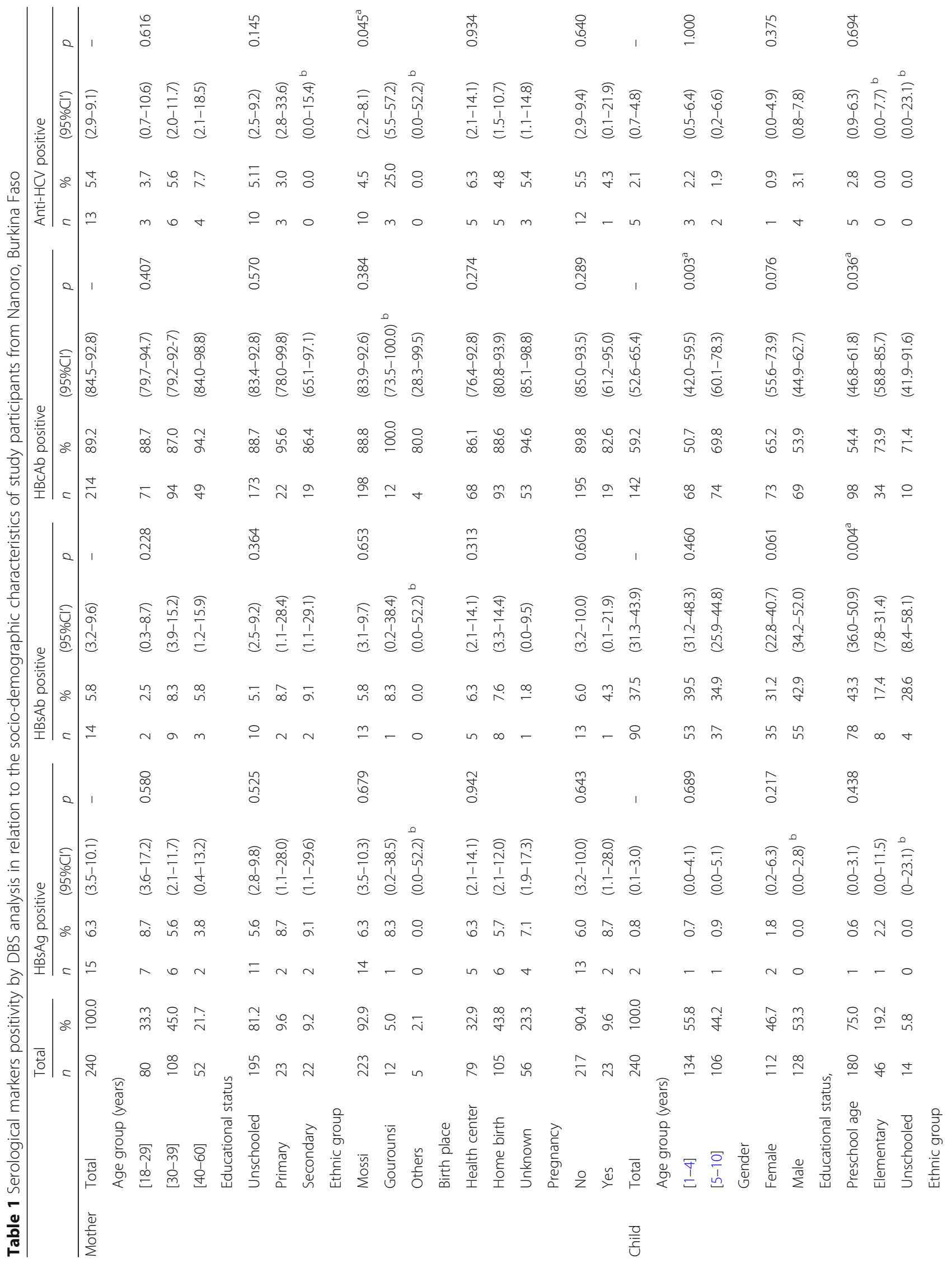




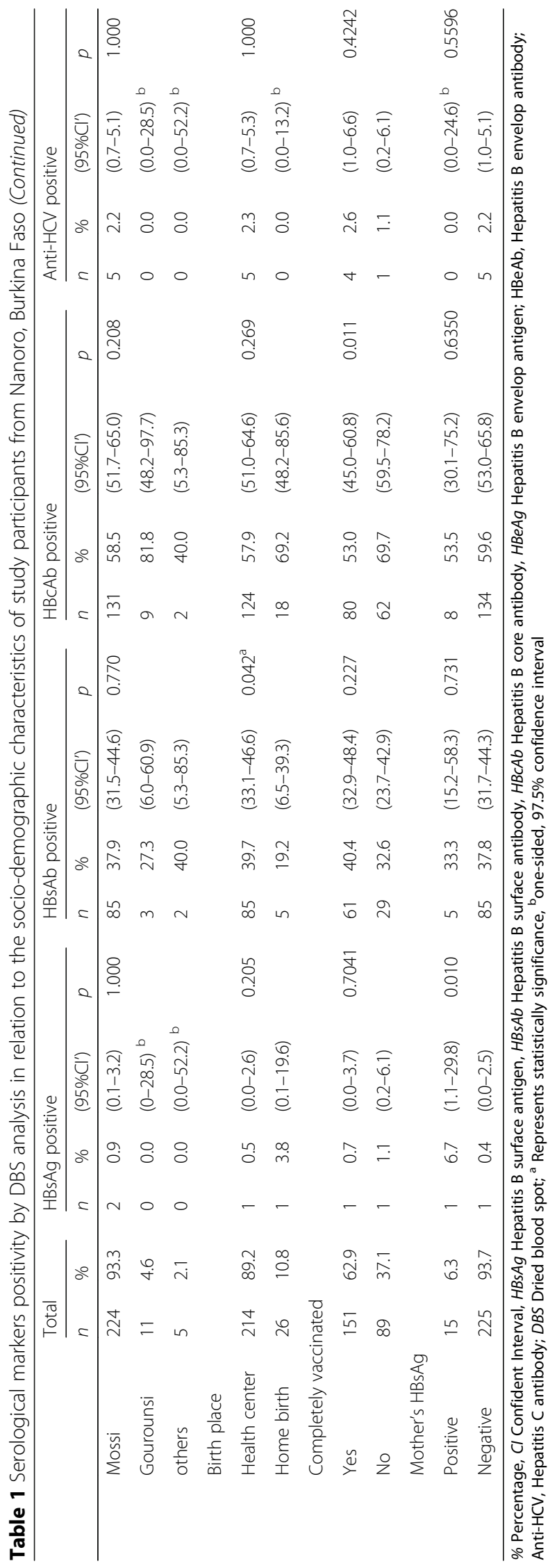




\section{a}
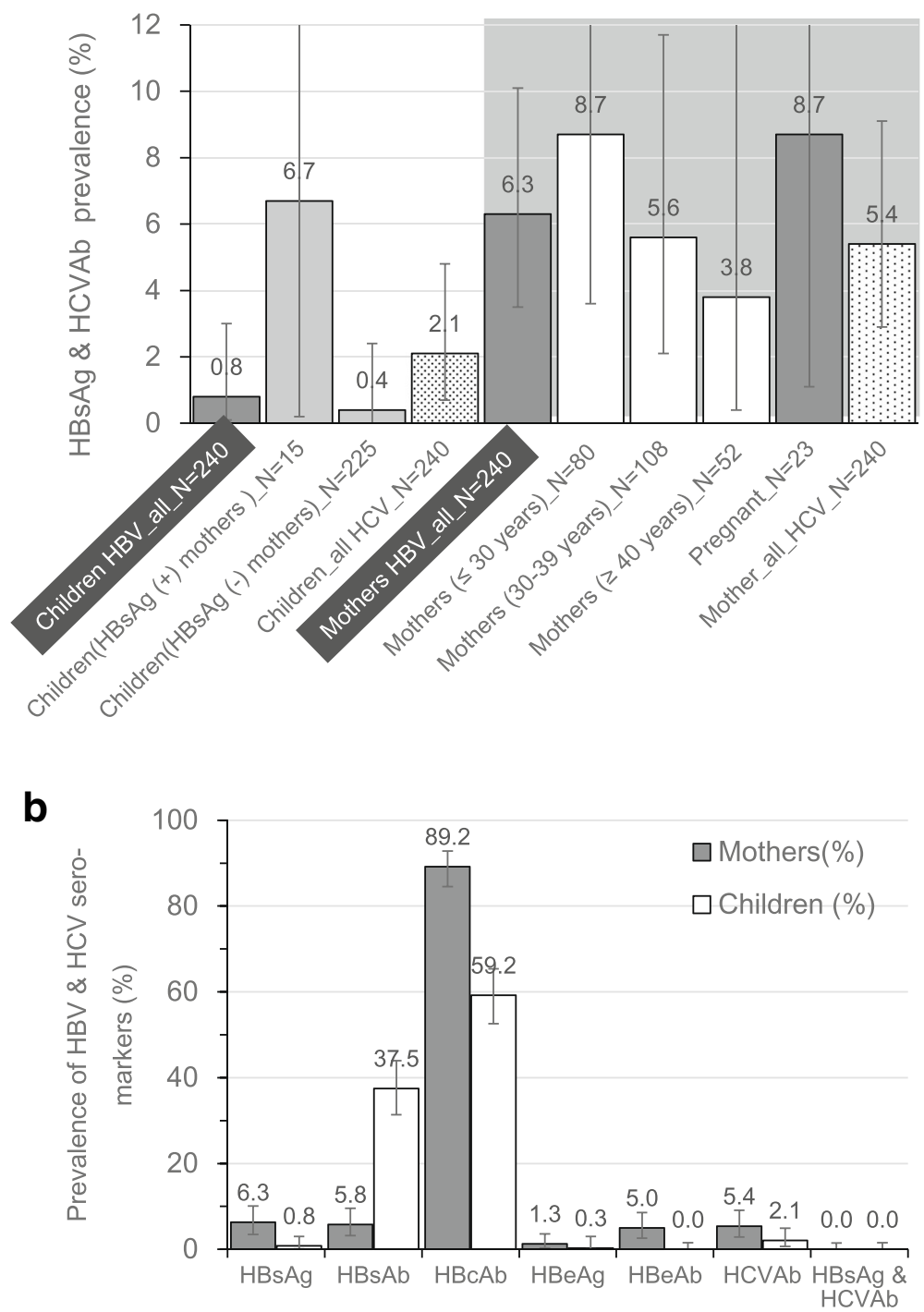

Fig. $1 \mathrm{HBsAg}$ and anti-HCV prevalence among mothers and children residing in Nanoro, rural area of Burkina Faso. a describes the extend of hepatitis B surface antigen and antibodies against hepatitis C virus among mothers and children assessed at the community level in Nanoro using dried blood spot samples collected on HemaSpot ${ }^{\mathrm{TM}}$. The white background presents data for the children and the dark background for the mothers. b describes the positive rates of each sero-marker of HBV (HBsAg, HBsAb, HBcAb, HBeAg, HBeAb) and HCV (HCVAb) for both mother and their children. The grey colour column represents the mothers and the while one represents the children

representative sample of women and their children. Burkina Faso is defined as a high transmission zone with HBsAg prevalence between $8.0-20.0 \%$, HBcAb between 70.0-95.0\% [22, 23]. Past studies have confirmed this assumption with HBsAg prevalence varying between 9.1 and $16.0 \%$ among adults with however data collected in the urban zone among specific populations including blood donors, pregnant women, HIV positive person, more likely to be at higher risk than the general population $[3,16,24]$. In this first study using a random sampling approach to assess all HBV infection seromarkers in rural Burkina Faso, a much lower HBsAg prevalence of $6.3 \%$ among adults mothers and $0.8 \%$ among children was reported. A higher prevalence among children (3.4\%) was reported in the western region and was even higher in its rural area (7.1\%) [25]. The lower HBsAg observed prevalence observe in the current study could be related to multiple interventions undertaken within the national viral hepatitis control program, among them the introduction of hepatitis $B$ vaccine into the national EPI schedule as well as the hepatitis $B$ vaccine clinical trials conducted in the area within the last decade. The impact of hepatitis $B$ vaccine in reducing the circulating HBsAg was already reported [26]. However, vaccine 


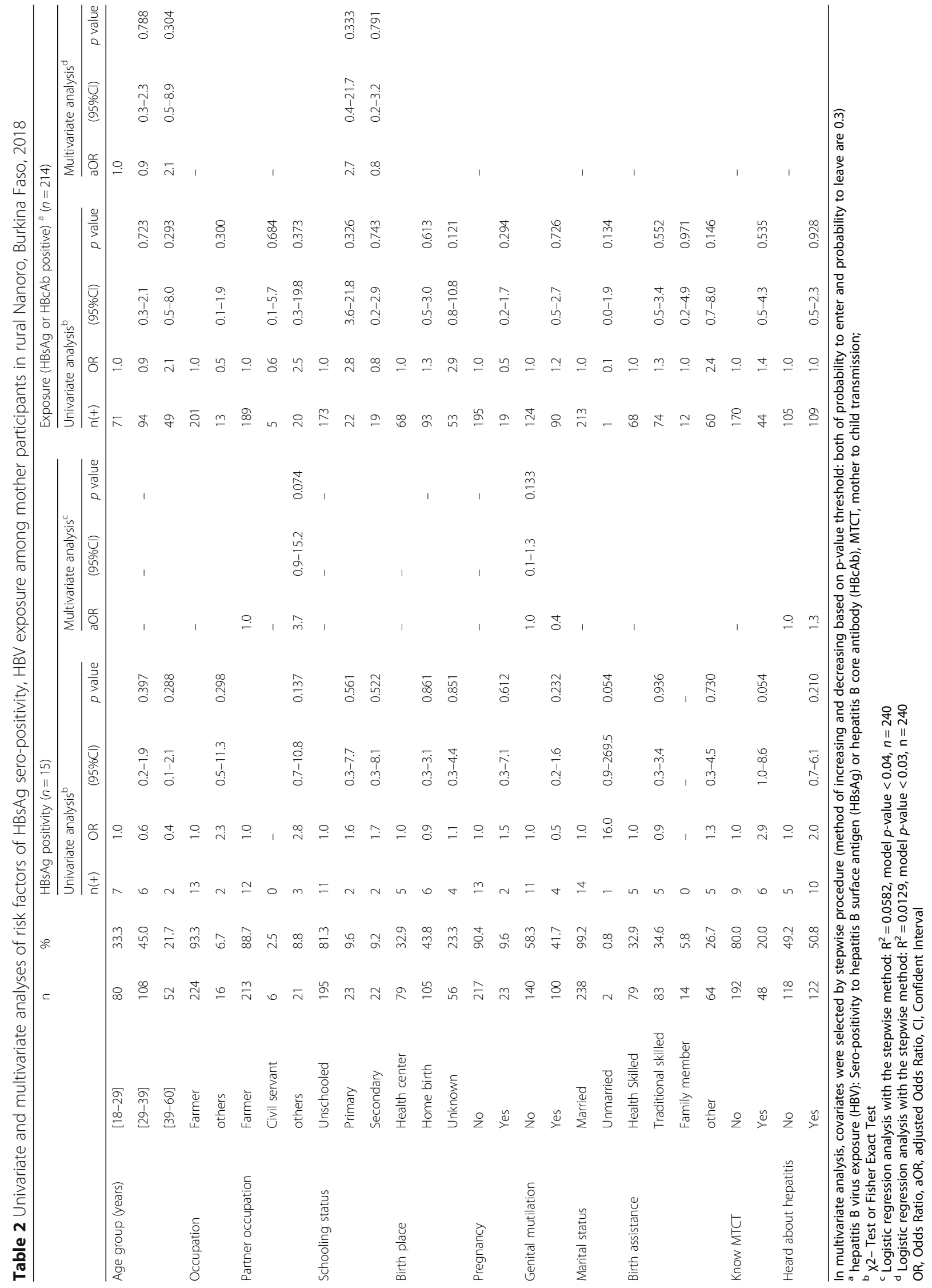




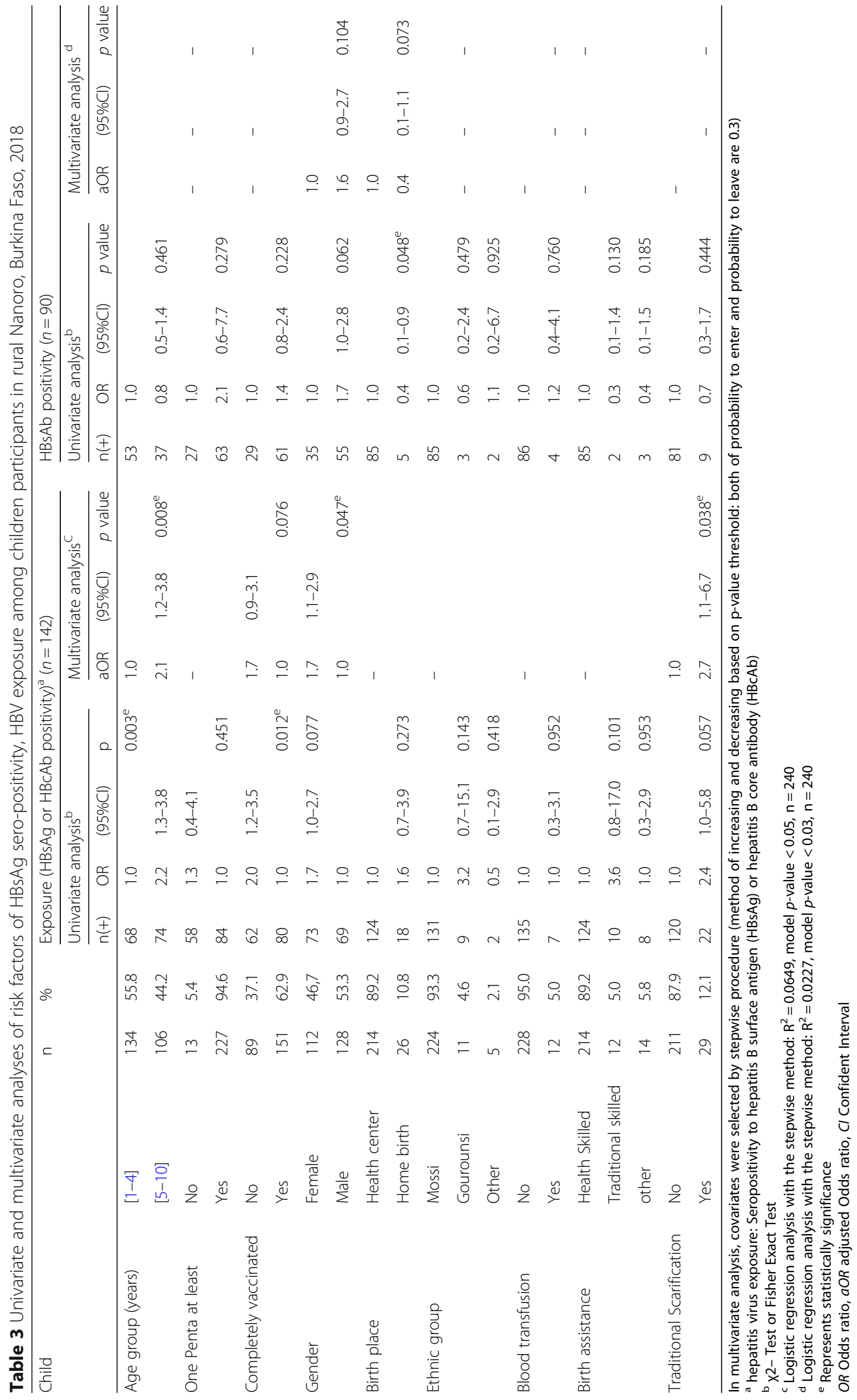




\section{HBV-Full length genome sequences}

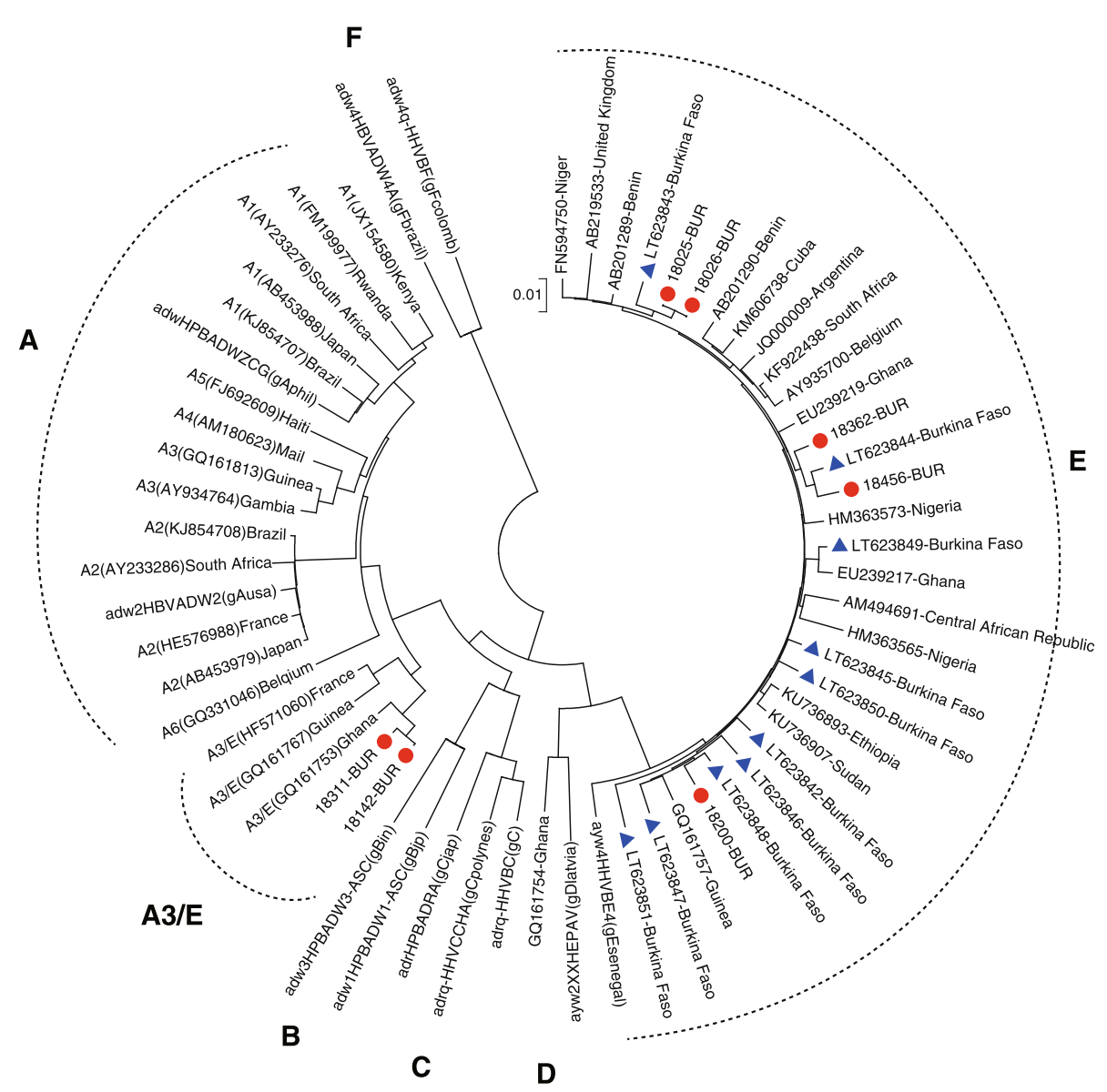

Fig. 2 Evolutionary relationships of taxa of HBVThe evolutionary history was inferred using the Neighbor-Joining method. The optimal tree with the sum of branch length $=0.83994352$ is shown. The tree is drawn to scale, with branch lengths in the same units as those of the evolutionary distances used to infer the phylogenetic tree. The evolutionary distances were computed using the Maximum Composite Likelihood method and are in the units of the number of base substitutions per site. The analysis involved 62 nucleotide sequences. All positions containing gaps and missing data were eliminated. There were a total of 3054 positions in the final dataset. Evolutionary analyses were conducted in MEGA7.

completion rate was not satisfactory in children with $63.0 \%$ completely vaccinated during their childhood. This is quite alarming as vaccination is free for children. Therefore, it is urgent to improve awareness among population as only a small proportion of mothers $(11.7 \%)$ in our study knew that vaccine could protect against hepatitis infection. The rate of exposure significantly increased with the older age, and this could be related to a larger vaccine coverage in younger children. Indeed, hepatitis B vaccine coverage was significantly higher in under 5 years age children than their elder peers $(p<0.001)$ and advocates for the improvement of HBV vaccine coverage in children. Pregnant women and children born to positive HBsAg carrier mothers were more at risk of infection and advocates the necessity to improve prevention in pregnant women and their newborn through either the administration of hepatitis B vaccination birth dose or by adequately diagnosing and treating high risk mothers during pregnancy to prevent transmission to their babies [27]. Indeed, pregnant women, particularly those with high viral load and positive HBeAg test have higher risk of passing the infection to their new-born during delivery [28]. Furthermore, it is known that high proportion of children infected at birth eventually evolve to chronicity, and birth dose is shown to reduce up to $90 \%$ of this risk [29,30]. Screening and treating pregnant women as well as birth dose vaccine, are key to achieving the national target.

Very good agreement between CLEIA and the Alere Determine $^{\text {Tn }}$ HBsAg rapid test was reported in this study and suggest that this rapid test could be advocated for screening hepatitis B infection in resource limited setting where good laboratory facilities are limited. 


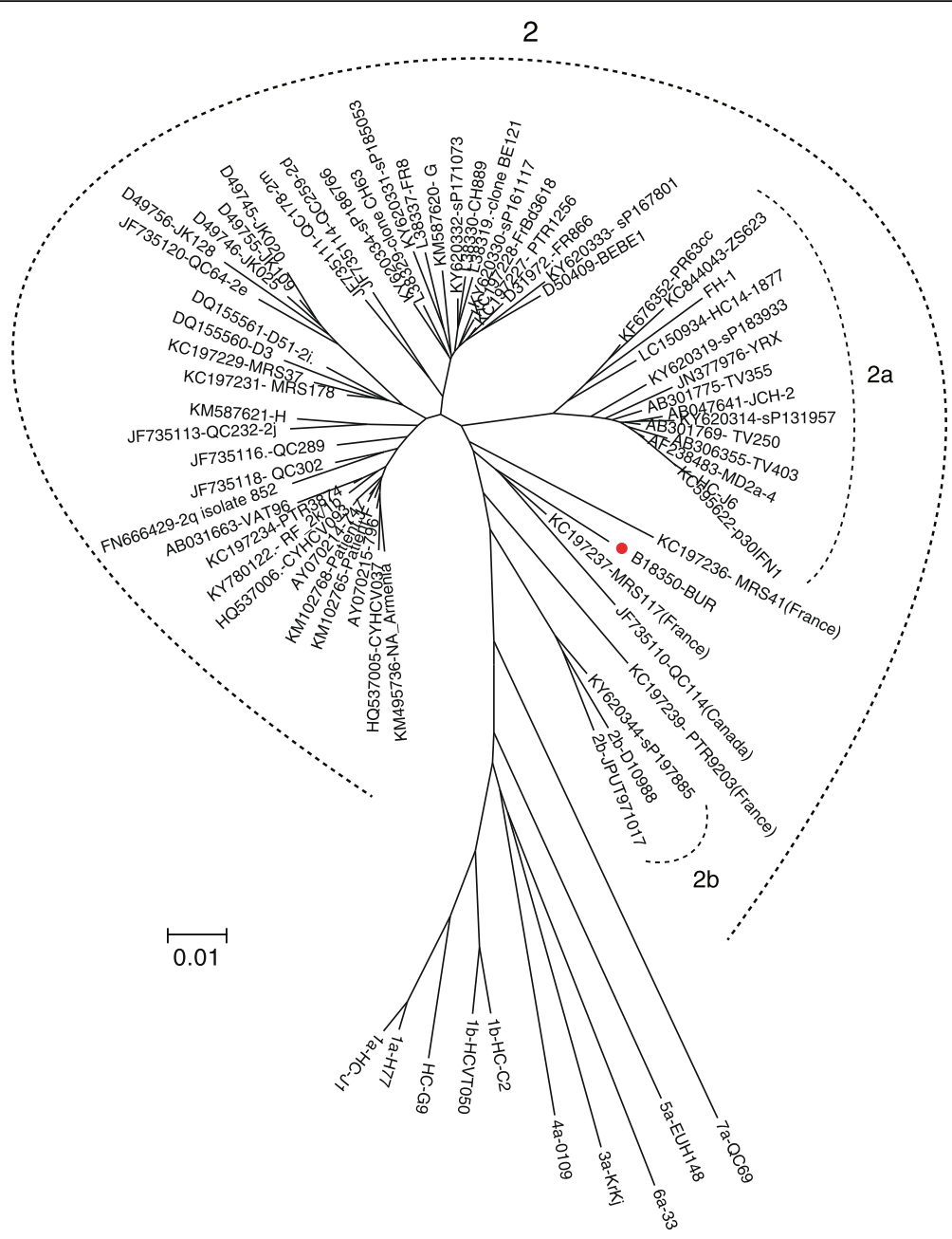

Fig. 3 Evolutionary relationships of taxa of HCV. The evolutionary history was inferred using neighbor-joining method

HBV DNA was detected in $65.0 \%$ of HBsAg positive samples with high viral load (>5 log IU/ mL) estimated in 5 cases. Phylogenetic analysis reported 100\% homology between case 18,025-BUR (child) and case 18,026BUR (mother) and this imply the persistence of mother to child vertical transmission. The mother of the other child case (18311-BUR) was HBsAg negative, and therefore classified as horizontal transmission. Among 240 mother and child pairs in this study, one pair is evident to be infected through mother-to-child vertical transmission $(0.42 \%)$ and the rest one is infected through horizontal transmission $(0.42 \%)$ possibly from the household contacts. Sequence comparison in GenBank shows a new recombinant genotype and might have an implication in the clinical manifestation and treatment approaches. Here by this study, the rate of mother-to-child transmission of HBV infection showed very low percentage $(<1 \%)$ which indirectly proves the effectiveness of hepatitis $B$ vaccination to all infant as national immunization program in Burkina Faso. The similar study in Cambodia also showed the effectiveness of hepatitis $\mathrm{B}$ vaccination including hepatitis $\mathrm{B}$ birth dose vaccine within $24 \mathrm{~h}$ after delivery which significantly reduce HBsAg prevalence to less than $1 \%$ in post-vaccine cohort of 5-7 years old children [31].

In January 2006, the government of Burkina Faso introduced hepatitis B vaccine as free immunization for children. However, among the $37.5 \%$ of children with protective level of $\mathrm{HBsAb}, 60.0 \%$ were secondary to past exposure to the wild type of the virus. The reasons behind this could be an inadequate timing of HBV vaccination in a region with high risk of infection, and the low quality of available doses due to multiples reasons including the challenges in maintaining adequate cold chain [32], sub-quality of vaccine dose available, individual variation in immune response or a poor vaccine coverage. The low response rate reported in the current study contrasts with results reported in others studies conducted in the country. Indeed, Kissou et al [33], and Ouedraogo et al [34], among under 3 years age children 
all reported approximately $90 \%$ response rates. The reasons behind these high rates could be related to the fact they did not discriminate participant immune due to vaccine or past infection, and also the timing between vaccine completion and their analyses. The study in Gambia documented that hepatitis B vaccine was $84 \%$ effective against infection and $94 \%$ effective against chronic carriage. But breakthrough infection and chronic carriage could occur more in vaccinated infants of mothers positive for hepatitis B surface and e antigens were than infants of uninfected mothers [35]. The study in Egypt also showed the existence of breakthrough infection despite of complete three dose hepatitis B vaccine with $57.2 \%$ of the sero protective rate after complete 3 doses hepatitis B vaccine [36]. In univariate analysis, serological vaccination rate was significantly low in children born at home than their peers born in health centres $(\mathrm{OR}=0.4,95 \% \mathrm{CI}, 0.1-0.9, p=0.048)$. Even though this was not significant in multivariate analysis, the trend was maintained $(\mathrm{aOR}=0.4,95 \% \mathrm{CI}$, $0.1-1.1, p=0.073$ ). This could be explained by the fact that mothers who delivered at home were less likely to visit health centres and then vaccinate their children. This hypothesis is supported by the significant low rate of well vaccinated children born at home compared to their counterpart born in health centres $(p=0.006)$. This stresses on the necessity to end home delivery in the rural area in order to improve vaccine effectiveness. This is in line with the WHO recommendation of promoting health centre delivery for all women. Awareness raising on the effects of home birth could help achieved this goal.

The prevalence of anti-HCV in this study was $5.4 \%$ in mothers and $2.1 \%$ among children. Meda et al. [16], found a similar prevalence $3.6 \%$, Zeba et al. [37], 4.4\% among blood donors in 2013. This is indicative of a stagnant prevalence of hepatitis $C$ infection in the country. Indeed, $\mathrm{HCV}$ was long ignored as public health problem in the country and was therefore given little attention. With the availability of high effective DAAs in the country, more interventions should be initiated. Anti-HCV positivity was significantly higher in participant with history of blood transfusion; however risk analysis did not show significant association as described elsewhere in a Cambodian study [38]. The reasons behind this finding may include a few populations with history of transfusion in the current study. A larger sample size could provide more details regarding blood safety and risk factors, to guide future interventions.

\section{Conclusions}

In this study, serological analysis suggested that the carriage rate of HBsAg is gradually decreasing in rural Burkina Faso and 2020 WHO African region of HBsAg prevalence under $2 \%$ target in children under 5 years age is already met. In risk analysis, factors including older age, being female and traditional scarification positively contributed to the occurrence of the infection and need to be considered in policy formulations. Moreover, residual humoral immunity rate was not satisfactory in the children group, and home birth was reported as the main contributing factor of poor vaccination coverage and immunity response. Full genome analysis suggests that mother-to-child transmission represent is persistent, highlighting the importance of preventive measures in pregnant women including screening and treatment as well as the administration of HBV birth dose in their new-born babies at delivery. Furthermore, HBV genotype $\mathrm{E}$ was predominant, followed by genotype $\mathrm{A}$ and a new recombinant genotype A3/E was reported for the first time in the country, and might modify the course of the infection and also jeopardize the effectiveness of current measures available in the country.

\section{Supplementary information}

Supplementary information accompanies this paper at https://doi.org/10. 1186/s12879-019-4731-7.

\section{Additional file 1. Questionnaires used in the study. Appendix 1a is questionnaire for children and appendix $1 \mathrm{~b}$ for mothers. These questionnaires were solely developed for this study and it mainly included the demographic information, vaccination status of the children, general knowledge on the hepatitis B virus infection and the available of vaccination. \\ Additional file 2. Serological profile among study participants in Nanoro health district area in 2018, Burkina Faso. This table describes the positive rates of HBV and HCV seromarkers classified by its infection status. \\ Additional file 3. Hepatitis B surface antigen ( $\mathrm{HBsAg}$ )-positive cases among mothers and their children in Nanoro, Burkina Faso, 2018. This table describes the complete information of the HBV strains detected from $\mathrm{HBsAg}$ positive mothers and their children, including seromarkers status, viral load, the genotypes and the length of sequences.}

\section{Abbreviations}

95\% Cl: 95\% exact confidence intervals; CLEIA: Chemiluminescent enzyme immunoassay; COI: Cut-off index; CRUN: Clinical research unit of nanoro; DAA: Direct antiviral agents; DBS: Dried blood spots; HBsAg: Hepatitis b surface antigen; HBV: Hepatitis b; HCV: Hepatitis c; HCVAb: Hcv antibody; HDSS: Health and demographic surveillance system area; NAT: Nucleic acid amplification test; OR: Odd-ratios; RNG: Random number generator; TBS: Trisbuffered saline

\section{Acknowledgements \\ We are indebted to the parents and children for their participation in this study. We acknowledge the valuable contribution of the Nanoro field staff, the data management team particularly Dr. Toussaint Rouamba, and the laboratory team for their support in managing the biological samples. We are grateful to Dr. Shunji Mishiro and the Viral Hepatitis Research Foundation of Japan for its invaluable help via the AAOHELP (Asia-Africa-Oceania Hepatitis Expert Linkage Promotion) project. Our special thanks go to Ms. Chiyoko Kuroda and Ms. Chitomi Aratani from the EIDCP laboratory, Hiroshima University for their support in facilitating all laboratory work.}

\section{Authors' contribution}

$\mathrm{ML}, \mathrm{SO}, \mathrm{ASu}$ and JT have conducted the study, conceptualized and written large parts of the manuscript. SO, BK, ZT, PRB, TN, ASo, HS, KD and HT have contributed substantially to the expert work from the clinical and laboratory 
view and made important comments to the manuscript. ML, TA, and JT performed the statistical work and contributed to the conceptualizing and writing of the manuscript. SN, KK, and CY performed the laboratory analysis. $\mathrm{KT}$ supervised on the laboratory works and performed the genotyping. All authors read and approved the final manuscript.

\section{Funding}

This study was funded in part by the JSPS Rompaku program, the department of epidemiology, infectious diseases control and prevention of the Hiroshima University and also the clinical research unit of Nanoro in Burkina Faso. The funder of the study had no role in the study design and data collection, analysis, and interpretation of data and in writing the manuscript.

\section{Availability of data and materials}

The dataset used and analysed during the current study is available from the corresponding author on reasonable request. All sequenced data of Burkina Faso HBV isolates are registered at GenBank via DDBJ. The sequences can be accessed at http://getentry.ddbj.nig.ac.jp/top-e.html with accession number of LC513651-LC513657 for HBV and LC513766 for HCV. If any trouble is had accessing the data, the sequences are available from the corresponding author upon reasonable request.

\section{Ethics approval and consent to participate}

The study protocol was approved by the ethics committee for epidemiological research of Hiroshima University (Certificate E-1257) and the ethics committee of the ministry of health in Burkina Faso (Deliberation CERS-2018-02-023). All participants signed an informed consent before they can enter the study. Participants tested positive to hepatitis infection were referred to a specialized centre.

\section{Consent for publication}

Not applicable.

\section{Competing interests}

The authors declare that they have no competing interests.

\section{Author details}

${ }^{1}$ Department of Epidemiology, Infectious Disease Control and Prevention, Hiroshima University Graduate School of Biomedical and Health Sciences, 1-2-3 Kasumi, Minami-ku, Hiroshima 734-8551, Japan. ${ }^{2}$ Institut de Recherche en Sciences de la Santé, Direction Régionale du Centre Ouest (IRSS/DRCO), Nanoro 11 BP 218, Burkina Faso. ${ }^{3}$ Unité de Recherche Clinique de Nanoro (URCN), Nanoro 11 BP 218, Burkina Faso. ${ }^{4}$ Institut de Recherche en Sciences de la Santé, Bobo-Dioulasso, Burkina Faso.

\section{Received: 29 August 2019 Accepted: 23 December 2019}

\section{Published online: 15 January 2020}

\section{References}

1. World Health Organization (WHO). Global health sector strategy on viral hepatitis 2016-2021. Geneva, Switzerland; 2016. p. 56. https://www.who.int/ hepatitis/strategy2016-2021/en/. Accessed 6 Apr 2019.

2. World_Health_Organization. Global hepatitis report, 2017; 2017.

3. Lingani M, Akita T, Ouoba S, et al. High prevalence of hepatitis B infections in Burkina Faso (1996-2017): a systematic review with meta-analysis of epidemiological studies. BMC Public Health. 2018;18(1):551.

4. World_Health_Organization. Hepatitis. WHO regional Office for Africa; 2017. https://www.afro.who.int/health-topics/hepatitis. Accessed 6 Apr 2019

5. Villa E, Fattovich G, Mauro A, Pasino M. Natural history of chronic HBV infection: special emphasis on the prognostic implications of the inactive carrier state versus chronic hepatitis. Digest Liver Dis. 2011;43(Suppl 1):S8-14.

6. Perz JF, Armstrong GL, Farrington LA, Hutin YJF, Bell BP. The contributions of hepatitis $B$ virus and hepatitis $C$ virus infections to cirrhosis and primary liver cancer worldwide. J Hepatol. 2006;45(4):529-38.

7. Ghouri YA, Mian I, Rowe JH. Review of hepatocellular carcinoma: epidemiology, etiology, and carcinogenesis. J Carcinog. 2017;16:1-1.

8. WHO. Global Health Estimates 2016: Disease burden by Cause, Age, Sex, by Country and by Region, 2000-2016. 2018. http://www.who.int/healthinfo/ global_burden_disease/estimates/en/index1.html. Accessed 23 Sep 2018.
9. Chen SL, Morgan TR. The natural history of hepatitis $\mathrm{C}$ virus (HCV) infection. Int J Med Sci. 2006;3(2):47-52.

10. World Health Organization (WHO). Global health estimates 2016: disease burden by cause a, sex, by country and by region, 2000-2016; 2018. http:// www.who.int/healthinfo/global_burden_disease/estimates/en/index1.html. Accessed 23 Sep 2018

11. Otte im Kampe $\mathrm{E}$, Muller $\mathrm{O}$, Sie A, Becher H. Seasonal and temporal trends in allcause and malaria mortality in rural Burkina Faso, 1998-2007. Malar J. 2015;14:300.

12. Wiktor SZ, Hutin YJ. The global burden of viral hepatitis: better estimates to guide hepatitis elimination efforts. Lancet. 2016;388(10049):1030-1.

13. World_Health_Organization. Guidelines for the prevention, care and treatment of persons with chronic hepatitis B infection; 2015. http://www. who.int/hiv/pub/hepatitis/hepatitis-b-guidelines/en/. Accessed 20 Feb 2019

14. Asselah T, Marcellin P. Direct acting antivirals for the treatment of chronic hepatitis C: one pill a day for tomorrow. Liver Int. 2012;32(Suppl 1):88-102.

15. Abozeid M, Alsebaey A, Abdelsameea E, et al. High efficacy of generic and brand direct acting antivirals in treatment of chronic hepatitis C. Int J Infect Dis IJID. 2018;75:109-14.

16. Meda N, Tuaillon E, Kania D, et al. Hepatitis B and C virus seroprevalence, Burkina Faso: a cross-sectional study. Bull World Health Organ. 2018;96(11):750-9.

17. Derra K, Rouamba E, Kazienga A, et al. Profile: Nanoro health and demographic surveillance system. Int J Epidemiol. 2012;41(5):1293-301.

18. Lumipulse Fl, Japan. Report on HBsAg, HBsAb-N, HBcAb-N, HBeAg, HBeAb-N using Lumipulse G1200; 2017.

19. Minegishi K, Yoshikawa A, Kishimoto S, et al. Superiority of minipool nucleic acid amplification technology for hepatitis B virus over chemiluminescence immunoassay for hepatitis B surface antigen screening. Vox Sang. 2003;84(4):287-91.

20. Fujimoto M, Chuon C, Nagashima S, et al. A seroepidemiological survey of the effect of hepatitis $B$ vaccine and hepatitis $B$ and $C$ virus infections among elementary school students in Siem Reap province, Cambodia. Hepatol Res. 2018;48(3):E172-82.

21. Abe A, Inoue K, Tanaka T, et al. Quantitation of hepatitis B virus genomic DNA by real-time detection PCR. J Clin Microbiol. 1999;37(9):2899.

22. Teo E-K, Lok AS. Epidemiology, transmission, and prevention of hepatitis $B$ virus infection - UpToDate. 2016. https://www.uptodate.com/contents/ epidemiology-transmission-and-prevention-of-hepatitis-b-virus-infection. Accessed 12 Sept 2019.

23. Ott JJ, Stevens GA, Groeger J, Wiersma ST. Global epidemiology of hepatitis $B$ virus infection: new estimates of age-specific HBsAg seroprevalence and endemicity. Vaccine. 2012;30(12):2212-9.

24. Sanou AM, Benkirane $K$, Tinto B, et al. Prevalence of hepatitis B virus and hepatitis D virus coinfection in Western Burkina Faso and molecular characterization of the detected virus strains. Int J Infect Dis IJID. 2018;70:15-9.

25. Armel Moumouni S, Abdoul Kader I, Clément Ziemlé M, et al. Hepatitis B vaccination in Burkina Faso: prevalence of HBsAg carriage and immune response in children in the western region. J Infect Dev Countries. 2018; 12(11):1002-8.

26. Shepard CW, Simard EP, Finelli L, Fiore AE, Bell BP. Hepatitis B virus infection: epidemiology and vaccination. Epidemiol Rev. 2006;28:112-25.

27. Dwivedi M, Misra SP, Misra V, et al. Seroprevalence of hepatitis B infection during pregnancy and risk of perinatal transmission. Ind J Gastroenterol. 2011;30(2):66-71.

28. Keane E, Funk AL, Shimakawa Y. Systematic review with meta-analysis: the risk of mother-to-child transmission of hepatitis B virus infection in subSaharan Africa. Aliment Pharmacol Ther. 2016;44(10):1005-17.

29. Edmunds WJ, Medley GF, Nokes DJ, Hall AJ, Whittle HC. The influence of age on the development of the hepatitis B carrier state. Proc Biol Sci. 1993; 253(1337):197-201.

30. Cheung KW, Seto MTY, Wong SF. Towards complete eradication of hepatitis $B$ infection from perinatal transmission: review of the mechanisms of in utero infection and the use of antiviral treatment during pregnancy. Eur J Obstet Gynecol Reprod Biol. 2013;169(1):17-23.

31. Ork V, Woodring J, Shafiqul Hossain M, Wasley A, Nagashima S, Yamamoto C, et al. Hepatitis B surface antigen seroprevalence among pre- and postvaccine cohorts in Cambodia, 2017. Vaccine. 2019;37(35):5059-66.

32. Burstein $\mathrm{R}$, Dansereau $\mathrm{EA}$, Conner $\mathrm{RO}$, et al. Assessing vaccine cold chain storage quality: a cross-sectional study of health facilities in three African countries. Lancet. 2013;381:S25.

33. KS SAK, Bazie W, et al. Post-vaccine immunity against hepatitis B in Burkina Faso children. Gastroenterol Pancreatol Liver Disord. 2018:6(1):1-4. https:// doi.org/10.15226/2374-815X/6/1/001120. 
34. Ouedraogo HGKS, Tiendrebeogo S, Liestman B, Tarnagda G, Bationo F, et al. Immune and hepatitis B virus (HBV) infection status among children receiving hepatitis B immunization in Ouagadougou, Burkina Faso. J Pediatr Infect Dis. 2013;8(4):7.

35. Fortuin $M$, Chotard J, Jack AD, et al. Efficacy of hepatitis $B$ vaccine in the Gambian expanded programme on immunisation. Lancet. 1993; $341(8853): 1129-32$.

36. Salama II, Sami SM, Said ZNA, et al. Effectiveness of hepatitis B virus vaccination program in Egypt: multicenter national project. World I Hepatol. 2015;7(22):2418-26.

37. Zeba MTA, Sanou M, Bisseye C, et al. Characterisation of hepatitis C virus genotype among blood donors at the regional blood transfusion Centre of Ouagadougou, Burkina Faso. Blood Transfus. 2014;12 Suppl 1(Suppl 1):554-7.

38. Yamada $H$, Fujimoto $M$, Svay $S$, et al. Seroprevalence, genotypic distribution and potential risk factors of hepatitis $B$ and $C$ virus infections among adults in Siem Reap, Cambodia. Hepatol Res. 2015;45(4):480-7.

\section{Publisher's Note}

Springer Nature remains neutral with regard to jurisdictional claims in published maps and institutional affiliations.

Ready to submit your research? Choose BMC and benefit from:

- fast, convenient online submission

- thorough peer review by experienced researchers in your field

- rapid publication on acceptance

- support for research data, including large and complex data types

- gold Open Access which fosters wider collaboration and increased citations

- maximum visibility for your research: over $100 \mathrm{M}$ website views per year

At $\mathrm{BMC}$, research is always in progress.

Learn more biomedcentral.com/submissions 